Journal for ImmunoTherapy of Cancer

\title{
LAG-3: from molecular functions to clinical applications
}

\author{
Takumi Maruhashi (D) , Daisuke Sugiura (D) , Il-mi Okazaki (D) , Taku Okazaki
}

To cite: Maruhashi T, Sugiura D, Okazaki I, et al. LAG-3: from molecular functions to clinical applications. Journal for ImmunoTherapy of Cancer 2020;8:e001014. doi:10.1136/ jitc-2020-001014

Accepted 29 July 2020

Check for updates

(C) Author(s) (or their employer(s)) 2020. Re-use permitted under CC BY-NC. No commercial re-use. See rights and permissions. Published by BMJ.

Laboratory of Molecular Immunology, Institute for Quantitative Biosciences, The University of Tokyo, Tokyo, Japan

Correspondence to

Dr Taku Okazaki;

tokazaki@iqb.u-tokyo.ac.jp

\section{ABSTRACT}

To prevent the destruction of tissues owing to excessive and/or inappropriate immune responses, immune cells are under strict check by various regulatory mechanisms at multiple points. Inhibitory coreceptors, including programmed cell death 1 (PD-1) and cytotoxic T lymphocyte antigen 4 (CTLA-4), serve as critical checkpoints in restricting immune responses against self-tissues and tumor cells. Immune checkpoint inhibitors that block PD-1 and CTLA-4 pathways significantly improved the outcomes of patients with diverse cancer types and have revolutionized cancer treatment. However, response rates to such therapies are rather limited, and immune-related adverse events are also observed in a substantial patient population, leading to the urgent need for novel therapeutics with higher efficacy and lower toxicity. In addition to PD-1 and CTLA-4, a variety of stimulatory and inhibitory coreceptors are involved in the regulation of T cell activation. Such coreceptors are listed as potential drug targets, and the competition to develop novel immunotherapies targeting these coreceptors has been very fierce. Among such coreceptors, lymphocyte activation gene-3 (LAG-3) is expected as the foremost target next to PD-1 in the development of cancer therapy, and multiple clinical trials testing the efficacy of LAG-3-targeted therapy are underway. LAG-3 is a type I transmembrane protein with structural similarities to CD4. Accumulating evidence indicates that LAG3 is an inhibitory coreceptor and plays pivotal roles in autoimmunity, tumor immunity, and anti-infection immunity. In this review, we summarize the current understanding of LAG-3, ranging from its discovery to clinical application.

\section{INTRODUCTION}

Immune cells can rapidly activate powerful defense mechanisms when they encounter invading pathogens. However, excessive and/or undesirable immune responses can exert deleterious effects. Immune cells are regulated by various molecules and cells with suppressive functions at multiple checkpoints. Such checkpoints are critical to the development of self-tolerance as the immune cells learn not to attack host cells. However, such checkpoints can be hijacked by tumors and pathogens to escape from the immune system.

Cancer immunotherapies targeting inhibitory coreceptors programmed cell death 1
(PD-1) and cytotoxic T lymphocyte antigen 4 (CTLA-4) significantly improved the outcomes of patients with diverse cancer types, revolutionizing cancer treatment. The success of these therapies verified that inhibitory coreceptors serve as critical checkpoints for immune cells to not attack the tumor cells as well as self-tissues. However, response rates are typically lower and immune-related adverse events (irAEs) are also observed in patients administered with immune checkpoint inhibitors. This is indicative of the continued need to decipher the complex biology of inhibitory coreceptors to increase response rates and prevent such unwanted side effects in patients with cancer. ${ }^{1-5}$

To date, many stimulatory and inhibitory coreceptors have been identified in addition to PD-1 and CTLA-4. These coreceptors are supposed to control the activation of lymphocytes by regulating the quality and quantity of the antigen receptor signaling to optimize beneficial immune responses while avoiding autoimmunity and excess immune responses. There is an intense competition among pharmaceutical companies to develop novel immunotherapies targeting these coreceptors. Among such coreceptors, lymphocyte activation gene-3 (LAG-3, CD223) is the foremost target next to PD-1, and multiple clinical trials to validate LAG-3-targeted therapies are ongoing. ${ }^{6}$

\section{MOLECULAR CHARACTERISTICS OF LAG-3}

LAG-3 was identified in 1990 by Triebel and colleagues ${ }^{8}$ in a screening designed to isolate molecules that were selectively expressed in $\mathrm{F}_{5}$ cells, a CD3-negative interleukin (IL)-2-dependent NK cell line. They screened the cDNA library of $\mathrm{F}_{5}$ cells with a probe obtained by subtraction of the cDNA of $F_{5}$ cells with the mRNA of Jurkat ( $T$ cell leukemia), Laz 388 (Epstein-Barr virustransformed B lymphoblastoid cell), K562 (erythro-myeloid leukemia), and U937 (cell of histiocytic origin) cells. The LAG-3 cDNA 


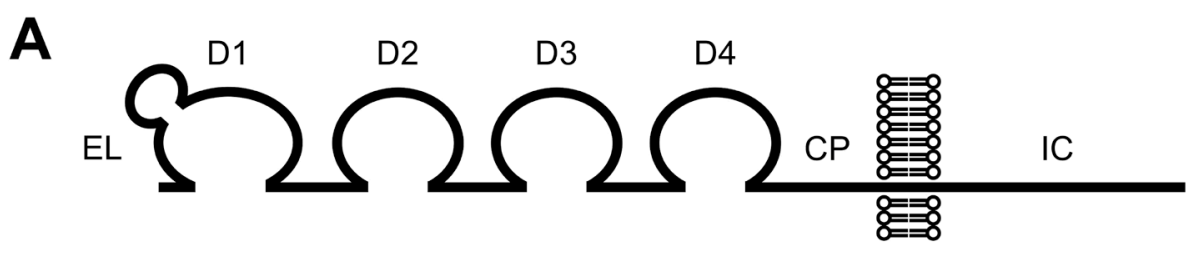

B

Human PPAAAPGHPLAPGPHPAAPSSWGPRPRRY Mouse QPTPIPALDL----HQGMPSPRQPAPGRY

Rat QPASIPALDLLQG----MPSTRRHPPHRY

Rabbit PPA---------------PAARGSGPQRY

Chicken -PQE-----L----H-------------H

Lizard ---LYQGLTLRWVRHEG--SS--HR-KRR

Whale -PAA--------------LSARGSGPRRY
C

Human ELSSPGAQRSGRAPGALPAGH

Mouse E-SSSGAHSARRISGDLKGGH

Rat E-SSSGAWSAKRISGDLKGGH

Rabbit GSPRPGARRSGRAPGALKAP-

Chicken QIT--GAQVSG--PPTIFSGQ

Lizard EITA--AQVSSSQPGT--PGK

Whale ELSRPGAQRSGRAPGAWKTGH

D

Human RRQWRPRRFSALEQGIHPPQAQSKIEELEQEPEPEPEPEPEPEPEPEPEQL

Mouse RKOLLLRRFSALEHGIQPFPAQRKIEELERELETEMGQEPEPEPEPQLEPEPRQL

Rat RRQLLRRRFSALEHGIRPPPVQSKIEELEREPETEMEPETEPDPEPQPEPELEPESRQL

Rabbit RROWRPRRFSALEHGAPPPOAOSKIEELELEAEAELEPEPGLEPQOEPROL

Chicken KRARLPASFPALERIVAVTVP--KEMEESOKETIQQTEC

Lizard RKRVTSSNFPALELMVAATLPG-KKEKVGDPEEKVQQMEY

Whale RRWWRPRRFSALEHETHPPQAQSKIEDPEPOLKOP

$$
\text { FxxL KIEELE EX-repeat }
$$

Figure 1 Structure of LAG-3. (A) Schematic representation of LAG-3. CP, connecting peptide; D1-D4, domains 1-4; EL, extra loop; IC, intracellular. (B-D) Alignments of EL, CP, and IC. Amino acid sequences of EL (B), CP (C), and IC (D) are shown for indicated species. Amino acid residues conserved between human and mouse are colored in red for $E L(B)$ and $C P(C)$. Putative FxxL, KIEELE, and EX-repeat are boxed. Amino acid sequences of LAG-3 were retrieved from Ensembl.org.

was isolated from $120 \mathrm{cDNA}$ clones that were obtained by the screening.

LAG-3 is a type I transmembrane protein with four Ig-like domains termed domain 1 (D1) to domain 4 (D4) (figure 1A). The extracellular region of LAG-3 shares approximately $20 \%$ amino acid homology with that of CD4, which is also composed of four Ig-like domains. In addition, the LAG-3 gene is located adjacent to the CD4 gene in most species (eg, on chromosome 12 and 6 in human and mouse, respectively). Hence, it is likely that these genes have evolved by gene duplication. Contrary to the similarity in the extracellular regions, the intracellular regions of LAG-3 and CD4 bear no noticeable similarity. LAG-3 lacks the cysteine motif required for the association with lymphocyte-specific protein tyrosine kinase (Lck) and the palmitoylation site observed in CD4. ${ }^{9-11}$ The organization of the genomic regions of CD4 and LAG-3 containing exons encoding their extracellular regions is similar, but the genomic organization containing exons encoding their intracellular regions is varied. Thus, CD4 and LAG-3 are closely related but are poised to exhibit divergent functions.

D1 of LAG-3 consists of nine $\beta$-strands that are assigned to the A, B, C, C', C", D, E, F, and G strands of the IgV fold. An additional sequence of about 30 amino acids is located between the C and C' strands, which forms a loop and is termed 'extra loop'. Although the sequences share low similarity, this loop can be observed in both human and mouse LAG-3 and is reported to engage in the association between LAG-3 and major histocompatibility complex class II (MHCII) (figure 1B). ${ }^{12}{ }^{13} \mathrm{CD} 4$ does not have an extra loop, and the mechanism of the contribution of the extra loop to the association between LAG-3 and MHCII is currently unknown. LAG-3 has been reported to be highly glycosylated, which is evident by the presence of multiple N-glycosylation sites in D2-D4. Galectin-3 and liver sinusoidal endothelial cell lectin (LSECtin) have been suggested to interact with glycans on LAG-3. ${ }^{14-16}$

A longer amino acid sequence termed 'connecting peptide' is located in the LAG-3 between D4 and the transmembrane region when compared with CD4. Based on the mouse model, Li et $a l^{17}$ reported that metalloproteinases a disintegrin and metallopeptidase domain (ADAM) 10 and ADAM17 cleaved LAG-3 at CP and released the extracellular region of LAG-3 in soluble form. LAG-3 mutants that can escape from the cleavage by metalloproteinases demonstrate stronger inhibitory effects. Hence, ADAM10 and ADAM17 presumably modulate the inhibitory effect of LAG-3 by regulating the amount of LAG-3 on the cell surface. The homology of the amino acid sequences of the CP between human and mouse is low. Whether human LAG-3 can also be cleaved by these metalloproteinases or not remains to be examined (figure 1C). In addition to the cleavage, soluble LAG-3 comprising D1-D3 can be produced by alternative splicing. Currently, the function of soluble LAG-3 is unknown. ${ }^{718}$

\section{EXPRESSION OF LAG-3}

Like PD-1 and CTLA-4, LAG-3 is not expressed on naive T cells, but its expression can be induced on $\mathrm{CD} 4^{+}$and $\mathrm{CD} 8^{+}$ $\mathrm{T}$ cells upon antigen stimulation. ${ }^{8}{ }^{19}$ As the inhibitory 
function of LAG-3 strongly correlates with its expression levels on the cell surface, ${ }^{20}$ the regulation of LAG-3 expression is very critical. Continuous antigen exposure owing to the chronic infection with viruses, ${ }^{21} 22$ bacteria, ${ }^{23}$ and parasites ${ }^{24}$ leads to high and sustained expression of LAG-3 as well as other inhibitory coreceptors on $\mathrm{CD}^{+}$ and $\mathrm{CD}^{+} \mathrm{T}$ cells. These $\mathrm{T}$ cells lose robust effector function and are termed exhausted T cells. LAG-3 blockade has been demonstrated to reinvigorate exhausted T cells and strengthen anti-infection immunity, although to a lesser extent compared with that by PD-1 blockade. ${ }^{22}$ 24-30 Tumor-infiltrating $\mathrm{T}$ cells are also persistently exposed to tumor-associated antigens and express high levels of multiple inhibitory coreceptors including LAG-3, resulting in functional exhaustion. ${ }^{31-36}$ IL-2, IL-7, and IL-12, but not IL-4, IL-6, IL-10, tumor necrosis factor (TNF), and interferon (IFN)- $\gamma$, have been reported to augment LAG-3 expression on activated T cells. ${ }^{37} 38$

LAG-3 expression is also detected on several subsets of $\mathrm{CD}^{+} \mathrm{T}$ cells with suppressive function. Foxp $3^{+}$regulatory $\mathrm{T}\left(\mathrm{T}_{\text {reg }}\right)$ cells constitutively express LAG- $3,{ }^{39}$ presumably due to the continuous activation of $\mathrm{T}$ cell receptor (TCR) signal by self-antigens, which is required for the differentiation, homeostasis, and suppressive functions of $\mathrm{T}_{\text {reg }}$ cells. ${ }^{40}$ Zhang et $a l^{41}$ demonstrated that LAG-3 on $\mathrm{T}_{\text {reg }}$ cells limited their proliferation intrinsically. The role of LAG-3 in the effector function of $\mathrm{T}_{\text {reg }}$ cells is contentious. Huang et $a l^{39}$ reported that $\mathrm{T}_{\text {reg }}$ reglls from LAG-3deficient mice inhibited the activation of effector $\mathrm{T}$ cells with lower efficiency whereas other studies demonstrated that the suppressive function of LAG-3-deficient $T_{\text {reg }}$ cells is comparable. ${ }^{1942}{ }^{43}$ Further studies are required to elucidate the actual role of LAG-3 in association with the $\mathrm{T}_{\text {reg }}$ cells. LAG-3 is also expressed on $\mathrm{CD}^{+}$type $1 \mathrm{~T}$ regulatory (Tr1) cells. Although Tr1 cells demonstrate strong immunosuppressive activity by secreting high amount of IL-10, specific cell surface markers that define this population had not been detected until they were observed to express LAG-3 and CD49b. ${ }^{44}$ In addition, LAG-3expressing $\mathrm{CD}^{+} \mathrm{CD}^{-} 5^{-}$Foxp $^{-} \mathrm{T}$ cells that produce IL-10 and transforming growth factor (TGF)- $\beta 3$ have been proposed to exhibit regulatory function as a distinct cell population. ${ }^{4546}$ Interestingly, IL-10-producing natural regulatory plasma cells have also been observed to express LAG- $3 .{ }^{47}$ Currently, the roles of LAG-3 in the cell-extrinsic inhibitory function of these non-classical regulatory cells remain unclear and require further investigation.

Multiple transcriptional regulators such as thymocyte selection-associated high mobility group box protein (TOX), nuclear factor of activated $\mathrm{T}$ cells (NFAT), nuclear receptor subfamily 4, group A (NR4A), interferon regulatory factor 4 , and $\mathrm{B}$ lymphocyte-induced maturation protein- 1 are known to engage in the generation of exhausted $\mathrm{T}$ cells. ${ }^{4-56}$ Among these, NFAT, NR4A, and TOX have been demonstrated to augment the expression levels of LAG-3, together with other inhibitory coreceptors, when overexpressed in T cells. Early growth response gene 2 (EGR2) is also reported to be a key transcription factor in the induction of LAG-3 expression in $\mathrm{CD} 4^{+} \mathrm{CD} 25^{-}$Foxp $^{-}$regulatory $\mathrm{T}$ cells. ${ }^{45}$ On the other hand, T-box expressed in T cells (T-bet) has been reported to repress the expression of LAG-3 and other inhibitory coreceptors and sustain antigen-specific response of $\mathrm{CD}^{+} \mathrm{T}$ cells during chronic infection. ${ }^{5758} \mathrm{In}$ addition to the transcriptional regulation, the cell surface expression level of LAG-3 is also regulated by subcellular trafficking and proteolytic cleavage. ${ }^{175960}$

LAG-3 is also expressed on $\mathrm{CD}^{+} \mathrm{CD}^{-} \mathrm{CD}^{-}{ }^{-} \mathrm{T}$ cells ${ }^{61}$ TCR $\alpha \beta C D 8 \alpha \alpha$ intraepithelial lymphocytes, ${ }^{62} \gamma \delta \mathrm{T}$ cells, ${ }^{6364}$ and NKT cells. ${ }^{65}$ Besides, its expression on activated NK cells is reported to be involved in the cytotoxicity against MHCI-negative target cells in mice. ${ }^{86}$ Plasmacytoid dendritic cells ${ }^{67}$ and activated B cells ${ }^{68}$ also express LAG-3 on their cell surface. However, the functional roles of LAG-3 in these populations remain poorly understood. Furthermore, LAG-3 is reported to be expressed on neurons and acts as a receptor for $\alpha$-synuclein fibrils. ${ }^{69}$

\section{LIGANDS OF LAG-3}

LAG-3 has been proposed to bind to MHCII with higher affinity than $\mathrm{CD} 4$ and inhibit $\mathrm{T}$ cell activation by interfering with the association of CD4 with MHCII. ${ }^{12}{ }^{64} 70$ However, LAG-3 has been demonstrated to inhibit $\mathrm{T}$ cell activation by a mechanism that is different from the competitive inhibition of $\mathrm{CD} 4$, and the discrepancy between the binding capacity of soluble LAG-3 protein and MHCII expression levels in cells has been recognized, making the actual ligand of LAG-3 elusive. ${ }^{19}{ }^{71}$ Recently, MHCII transactivator (CIITA) has been identified as a critical regulator of LAG-3 ligand. CIITA induces the expression of not only MHCII but also MHCII accessory molecules, including CD74 (invariant chain, Ii) and H2-DM. The MHCII accessory molecules contribute to the formation and the cell surface sorting of peptide-MHCII complex (pMHCII) exhibiting stable structural conformation in the conventional pathway of antigen presentation. ${ }^{72}$ LAG-3 distinguishes the conformation of pMHCII and selectively binds to stable pMHCII. Accordingly, LAG-3 preferentially inhibits the activation of $\mathrm{CD} 4^{+} \mathrm{T}$ cells that recognize stable pMHCII (figure 2A). ${ }^{71}$ It has also been demonstrated that LAG-3 does not compete with CD4 for pMHCII binding. ${ }^{71}$ Instead, LAG-3 inhibits T cell activation by transducing inhibitory signals via the intracellular region, as described in the next section.

As mentioned, LAG-3 is expressed on exhausted CD8 ${ }^{+}$ $\mathrm{T}$ cells in tumors and is a potent therapeutic target for cancer immunotherapy. However, the mechanism by which LAG-3 binding to pMHCII could impact the activation of $\mathrm{CD}^{+} \mathrm{T}$ cells has not been elucidated. Recently, the activation of $\mathrm{CD}^{+} \mathrm{T}$ cells has been demonstrated to be inhibited weakly when antigen-presenting cells express a substantial amount of stable pMHCII in addition to cognate pMHCI, indicating that LAG-3 can also directly suppress $\mathrm{CD}^{+} \mathrm{T}$ cells to a certain extent. ${ }^{71}$ 
A
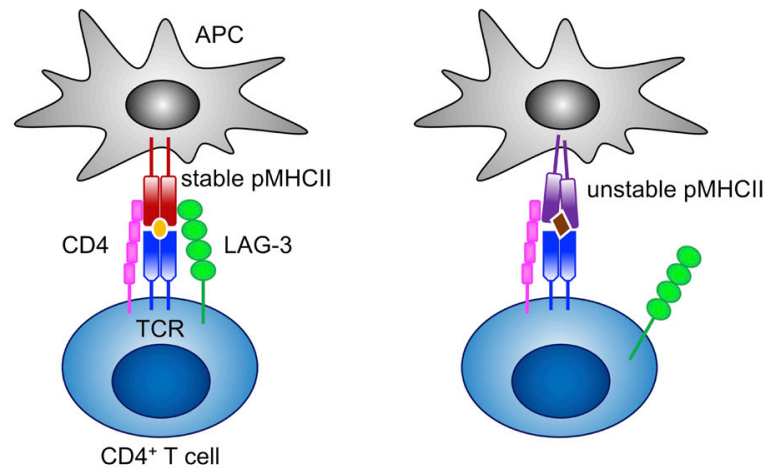

B

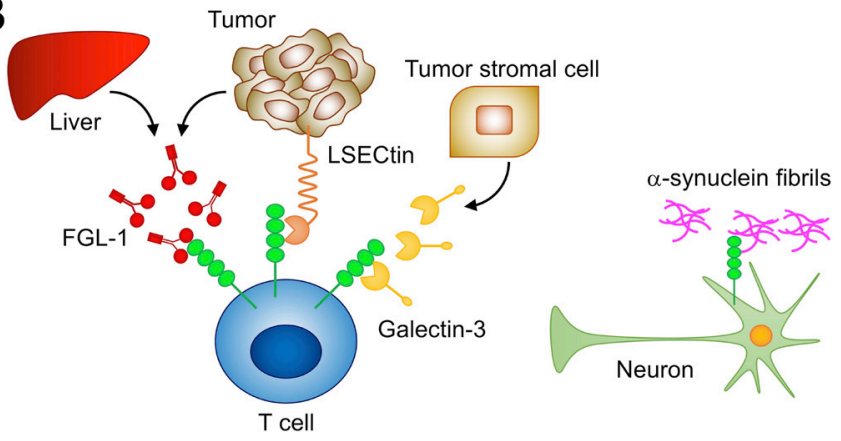

Figure 2 Ligands of LAG-3. (A) LAG-3 selectively binds to stable pMHCll and inhibits the activation of $\mathrm{CD}^{+}{ }^{+} \mathrm{T}$ cells that recognize stable pMHCII. (B) Reported non-MHCII ligands. LAG-3 has been reported to associate with FGL1, LSECtin, galectin-3, and $\alpha$-synuclein fibrils. FGL1, fibrinogen-like protein 1; LSECtin, liver sinusoidal endothelial cell lectin; MHCII, major histocompatibility complex class II; pMHCII, peptide-MHCII complex; APC, antigen presenting cell; TCR, T cell receptor.

To date, several molecules other than stable pMHCII have been reported as possible ligands for LAG-3 (figure 2B). As mentioned, galectin-3 and LSECtin have been indicated to interact with glycans on LAG-3. LSECtin is a member of the C-type lectin family and is expressed mainly in the liver. ${ }^{16} 73 \mathrm{Xu}$ et $a l^{16}$ reported that LAG-3 suppressed the IFN- $\gamma$ production from $\mathrm{T}$ cells upon stimulation with anti-CD3 Ab in the presence of LSECtin-expressing melanoma cells. Galectin-3, belonging to the galectin family, is a soluble galactose-binding lectin secreted from various types of tumor cells and tumor stromal cells. ${ }^{74}$ Kouo et $a l^{15}$ demonstrated that galectin-3 reduced the frequency of IFN- $\gamma$-producing $\mathrm{CD} 8^{+} \mathrm{T}$ cells upon stimulation with anti-CD3 and anti-CD28 Abs when $\mathrm{CD}^{+} \mathrm{T}$ cells from LAG-3-sufficient and not LAG-3-deficient mice were used. Recently, Wang $e t a l^{75}$ identified fibrinogen-like protein 1 (FGL1), a member of the fibrinogen family, as a potential ligand for LAG-3. FGL1 is secreted from hepatocytes in the liver under normal physiological conditions, whereas some tumor cells can also produce FGL1 at high levels. FGL1 has been demonstrated to reduce the secretion of IL-2 from 3A9 T hybridoma cells expressing LAG-3 upon stimulation with the cognate peptide. Further studies are required to elucidate whether and how these potential ligands independently and/or cooperatively contribute to the inhibitory function of LAG-3.

In addition to the immunoinhibitory role, LAG-3 seems to have a distinct role in the nervous system as well. Mao et $a b^{69}$ reported that LAG-3 can bind to $\alpha$-synuclein fibrils, which are associated with the pathogenesis of Parkinson's disease. The association of $\alpha$-synuclein fibrils with LAG-3 triggers endocytosis, cell-to-cell transmission, and neurotoxicity of $\alpha$-synuclein fibrils. In addition to LAG-3, other molecules such as semaphorins and paired Ig-like receptor $\mathrm{B}$ are known to exhibit dual roles in immune and nervous systems. ${ }^{76} 77$ Further studies are expected to demonstrate the similarities and discrepancies in the roles of such coreceptors between immune and nervous systems.

\section{INHIBITORY MECHANISMS OF LAG-3}

The intracellular region of LAG-3 consisting of approximately 60 amino acid residues lacks a typical inhibitory motif, such as immunoreceptor tyrosine-based inhibitory motif. However, it contains several amino acid sequences that are well conserved over different species of LAG-3 but are not shared with other inhibitory coreceptors. Such sequences include FSAL in the juxtamembrane region, KIEELE in the central region, and 10-15 tandem repeats of glutamate, and favorably but not limited to proline (EX-repeat) in the C-terminal region (figure 1D). As the intracellular region is required for LAG-3 to inhibit $\mathrm{T}$ cell activation, it can transduce distinct yet undetermined inhibitory signals via such sequences.

Workman $e \mathrm{al}^{13}$ reported that the lysine residue of the KIEELE sequence is required for the LAG-3-mediated inhibition of the antigen-dependent activation of 3A9 hybridoma T cells. However, a contradictory report has been published and the mechanisms of the contribution of lysine residue to the LAG-3-mediated inhibition have not been elucidated yet. ${ }^{20}$ Iouzalen $e t a l^{78}$ identified LAG-3-associated protein (LAP) bound to the EX-repeat of LAG-3 by employing yeast two-hybrid cloning experiment. To date, no follow-up study has been reported and the function of LAP remains elusive.

The inhibitory function of LAG-3 strongly correlates with its expression levels on the cell surface, and the amino acid substitutions and deletions substantially affect the expression levels of LAG-3. ${ }^{20}$ When the potency of the inhibitory effects of mouse LAG-3 mutants was evaluated relative to their expression levels in the antigendependent activation of DO11.10 T hybridoma cells, the substitutions of phenylalanine and leucine in the FSAL sequence to alanine reduced the inhibitory capacity of LAG-3 significantly. In addition, when mutations in the FSAL sequence were combined with the deletion of the EX-repeat, the inhibitory capacity of LAG-3 was completely lost similar to the LAG-3 mutant lacking the entire intracellular region. Interestingly, the deletion of EX-repeat alone does not affect the inhibitory capacity of LAG-3. These results demonstrate that LAG-3 likely transduces 
two independent inhibitory signals via the FxxL motif and EX-repeat, while the molecular mechanisms of these signals are still unknown. ${ }^{20}$ Further analyses are expected to delineate the precise molecular mechanisms by which such motifs independently or cooperatively regulate the signaling pathways in $\mathrm{T}$ cell activation.

\section{LAG-3 IN AUTOIMMUNITY}

Inhibitory coreceptors play critical roles in the establishment and/or maintenance of immune tolerance to self as represented by the spontaneous development of autoimmune diseases in mice deficient for PD-1 and CTLA-4. ${ }^{79-81}$ In addition, immune checkpoint inhibitors targeting PD-1 and CTLA-4 activate not only tumor-specific T cells but also self-reactive T cells to induce tissue toxicities, termed irAEs. ${ }^{82-84}$ Unlike PD-1 and CTLA-4, LAG-3 deficiency itself does not cause autoimmunity in non-autoimmuneprone mouse strains. However, genetic deletion or blockade of LAG-3 exacerbates type 1 diabetes (T1D) in non-obese diabetic (NOD) mice, an animal model of T1D. ${ }^{19}{ }^{85}$ LAG-3-deficient NOD mice demonstrate accelerated infiltration of autoreactive $\mathrm{CD} 4^{+}$and $\mathrm{CD} 8^{+} \mathrm{T}$ cells in islets compared with that in the age-matched LAG-3sufficient NOD mice. In contrast, NOD mice lacking the cell surface expression of LAG-3 on $\mathrm{T}_{\text {reg }}$ cells exhibit delayed onset and decreased incidence of T1D, which is attributed to the enhanced proliferation and function of $\mathrm{T}_{\text {reg }}$ cells in the absence of LAG-3. ${ }^{41}$ Mice with compound deficiency of LAG-3 and PD-1 develop lethal autoimmune myocarditis on BALB/c, C57BL/6, and B10.D2 backgrounds, indicating that LAG-3 acts synergistically with PD-1 to prevent autoimmunity. ${ }^{196}$

In addition, LAG-3 has been reported to mitigate the autoimmune symptoms in experimental autoimmune models. Jha $e t a l^{87}$ reported that LAG-3 deficiency or blockade increased the susceptibility to mercury ( $\mathrm{Hg})$ induced autoimmunity by inhibiting the induction of tolerance to $\mathrm{Hg}$ in $\mathrm{C} 57 \mathrm{BL} / 6 . \mathrm{H} 2^{\mathrm{s}}$ mice. In myelin oligodendrocyte glycoprotein (MOG)-induced experimental autoimmune encephalomyelitis (EAE) model, Kadowaki et $a l^{88}$ demonstrated that LAG-3 blockade abrogated the anti-inflammatory effect of gut environment-induced intraepithelial MOG-specific CD4 ${ }^{+} \mathrm{T}$ cells. Kim $e t a l^{89}$ also reported that the ability of in vitro-generated induced $\mathrm{T}_{\text {re }}$ $\left(\mathrm{i} \mathrm{T}_{\text {reg }}\right.$ ) cells to rescue $\mathrm{T}_{\text {reg }}$-depleted mice from lethal $\mathrm{EAE}$ was dependent on the expression of LAG-3 on the $\mathrm{iT}_{\text {reg }}$ cells.

Given the critical regulatory role of LAG-3 in autoimmunity, LAG-3 has been expected to be a promising therapeutic target in inflammatory and autoimmune diseases. A humanized anti-LAG-3 Ab with antibody-dependent cell cytotoxic activity (GSK2831781) has been developed to treat autoimmune diseases by eliminating LAG-3expressing $\mathrm{T}$ cells that presumably include pathogenic autoreactive T cells. ${ }^{90}$ In addition, agonistic anti-LAG-3 Ab (IMP761) has been reported to exert immunosuppressive effects both in vitro and in vivo by eliciting the inhibitory function of LAG-3 ${ }^{91}$

\section{LAG-3 IN ANTITUMOR IMMUNITY}

As mentioned, LAG-3 is expressed on exhausted $\mathrm{CD} 4^{+}$ and $\mathrm{CD}^{+}$tumor-infiltrating $\mathrm{T}$ cells that are defective in cytokine production. ${ }^{32-36}$ LAG-3 is also expressed on $\mathrm{T}_{\text {reg }}$ cells in the peripheral blood and tumor tissues of patients with melanoma, colorectal cancer, and non-small cell lung cancer. ${ }^{92} 93$ Such LAG-3-expressing $\mathrm{T}_{\text {reg }}$ cells produce high levels of immunoregulatory cytokines IL-10 and TGF- $\beta$ and suppress tumor-specific T cells. Consistently, the levels of LAG-3 expression and infiltration of LAG-3 ${ }^{+}$ cells in tumors have been reported to be associated with tumor progression, poor prognosis, and unfavorable clinical outcomes in various types of human tumors, such as colorectal cancer, ${ }^{94}$ renal cell carcinoma ${ }^{95}$ follicular lymphoma ${ }^{36}$ head and neck squamous cell carcinoma (HNSCC),${ }^{96}$ non-small cell lung cancer,${ }^{97}$ breast cancer, ${ }^{98}$ and diffuse large B cell lymphoma. ${ }^{99}$ These results strongly indicate that LAG-3 contributes to immune escape mechanisms in tumors similar to PD-1. Therefore, LAG-3 has been proposed as a promising therapeutic target for cancer immunotherapy, which is also supported by studies using animal models. Tumor growth delayed by anti-LAG-3 Ab has been reported in the mouse models of HNSCC and fibrosarcoma. ${ }^{96}{ }^{100}$ Grosso et al ${ }^{101}$ demonstrated that the combinatorial therapy incorporating anti-LAG-3 Ab and vaccination with tumor-associated antigen increased the number of activated $\mathrm{CD}^{+} \mathrm{T}$ cells in the tumor and disrupted the tumor parenchyma in the tumor-tolerance model of prostate cancer. However, no substantial difference in the grade of tumor was noted between mice administered with combination therapy or vaccination alone.

LAG-3 acts synergistically with PD-1 to suppress antitumor immunity as well as autoimmunity. In patients with epithelial ovarian cancer, Matsuzaki et $a \mathrm{l}^{34}$ observed that approximately $80 \%$ and $50 \%$ of $\mathrm{LAG}^{-} 3^{+}$and LAG-3 $3^{-}$tumor-infiltrating $\mathrm{CD} 8^{+}$ T cells expressed PD-1, respectively. They also reported that the co-blockade of LAG-3 and PD-1 augmented the proliferation and cytokine production of tumor-infiltrating $\mathrm{CD}^{+}$ $\mathrm{T}$ cells upon ex vivo stimulation with the tumor-associated antigen NY-ESO-1. Coexpression of LAG-3 and PD-1 on tumor-infiltrating $\mathrm{CD}^{+}$and $\mathrm{CD}^{+} \mathrm{T}$ cells and the strong therapeutic effect of co-blockade or compound genetic deletion of LAG-3 and PD-1 have also been observed in various mouse tumor models, such as B16 melanoma, MC38 colon adenocarcinoma, Sa1N fibrosarcoma, ovalbumin-expressing mouse epithelial ovarian cancer cell (IE9mp1), chronic lymphocytic leukemia derived from Em-TCL1 mice, and recurrent melanoma. ${ }^{86}{ }^{102-104}$ Although LAG-3 blockade is expected to activate tumor-specific $\mathrm{CD} 4^{+}$and/or $\mathrm{CD} 8^{+} \mathrm{T}$ cells in such studies, it may also increase the number of $\mathrm{T}_{\text {reg }}$ cells, since LAG-3 has been reported to limit the proliferation of $\mathrm{T}_{\text {reg }}$ cells. According to Goding et al, ${ }^{102} \mathrm{~T}_{\text {reg }}$ cells are rather reduced by the co-blockade of LAG-3 and PD-1 in the 
Table 1 Summary of LAG-3-targeted drugs under clinical trial

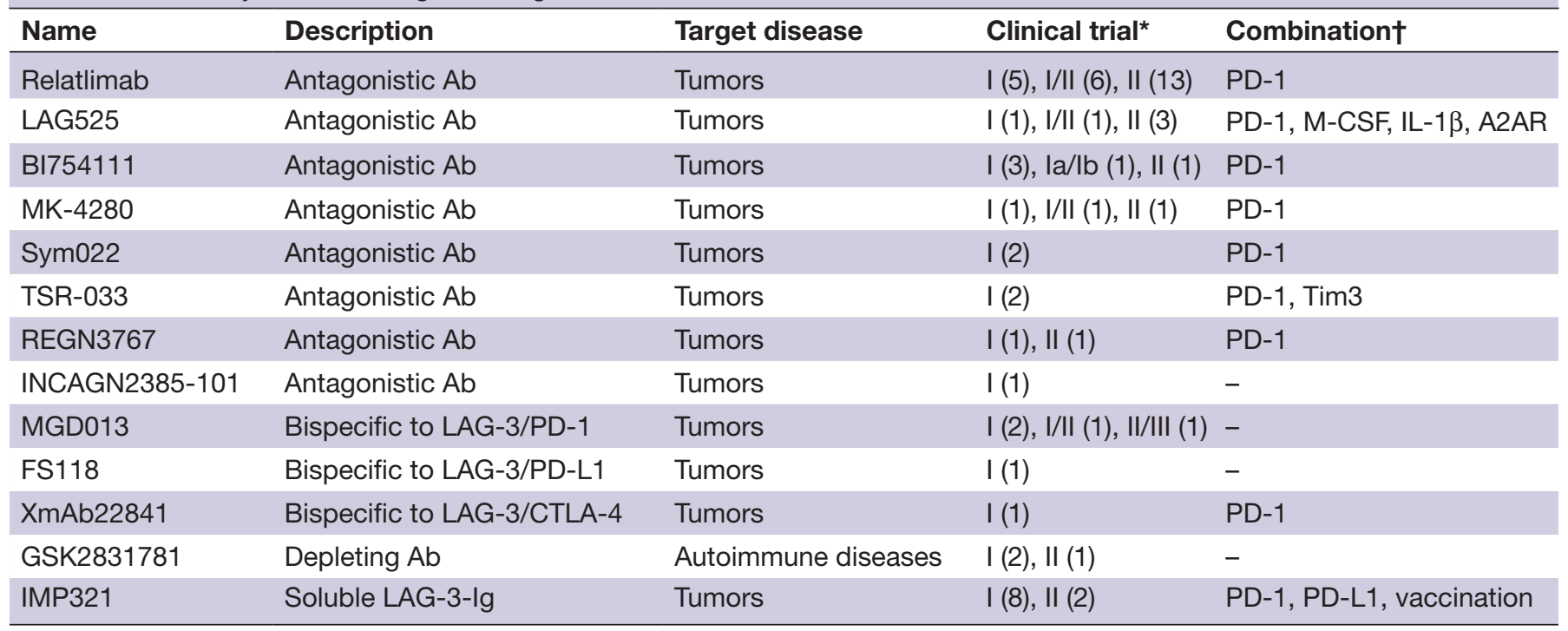

*The phase and the number of clinical trials listed on ClinicalTrials.gov are shown.

†Immune-related molecules targeted in combination therapies listed on ClinicalTrials.gov are shown.

A2AR, adenosine A2a receptor; CTLA-4, cytotoxic T lymphocyte antigen 4; IL-1 $\beta$, interleukin 1b; M-CSF, macrophage colony stimulating

factor; PD-1, programmed cell death 1; PD-L1, PD-1 ligand 1; Tim3, T-cell immunoglobulin and mucin-domain containing-3.

model of recurrence melanoma. Further studies are needed to delineate the exact effect of LAG-3 on the number and function of $\mathrm{T}_{\text {reg }}$ cells as well as effector $\mathrm{T}$ cells.

In most of these studies, anti-LAG-3 Ab resulted in modest therapeutic effects when used as monotherapy but markedly augmented the therapeutic effect of anti-PD-1 Ab. Differences in the inhibitory mechanisms and/or expression profiles of these two molecules most likely explain their functional synergy. Further studies are needed to comprehend the exact mechanisms of the synergistic or additive action of LAG-3 with PD-1.

\section{CLINICAL APPLICATION OF LAG-3}

Based on preclinical observations including those mentioned, agents that block or stimulate LAG-3 functions are expected to provide therapeutic benefits in the treatment of cancer or autoimmune diseases, especially when combined with agents targeting PD-1. To date, at least 13 agents that target LAG-3 have been developed (table 1). Anti-LAG-3 blocking Abs (relatlimab (BMS-986016), Sym022, TSR-033, REGN3767, LAG525, INCAGN2385-101, MK-4280, and BI754111) and antagonistic bispecific Abs (MGD013 (anti-PD-1/LAG-3), FS118 (anti-LAG-3/PD-L1), and XmAb22841 (anti-CTLA-4/ LAG-3)) are under clinical trials for various cancers either as monotherapy or in combination primarily with anti-PD-1 or anti-PD-L1 blocking Abs. According to the National Cancer Institute Drug Dictionary (https://www.cancer.gov/publications/dictionaries/cancer-drug), meeting abstracts, ${ }^{105-107}$ and a published report, ${ }^{108}$ most of them are supposed to block the interaction between LAG-3 and MHCII, while their effects on the interaction between LAG-3 and reported ligands other than MHCII are not specified.
To date, only a few interim reports of combinatorial therapies targeting LAG-3 and PD-1 are available. Future reports are awaited to observe the actual therapeutic efficacy of anti-LAG-3 Abs as monotherapy or the exact additive effects of anti-LAG-3 Abs in the combination therapy targeting PD-1 and LAG-3. In phase I/II study evaluating the safety and efficacy of relatlimab in combination with anti-PD-1 Ab (nivolumab) in patients with advanced melanoma that had progressed during previous anti-PD-1 or anti-PD-L1 immunotherapy (NCT0198609), the combination of relatlimab and nivolumab was well tolerated and the objective response rate (ORR) was $11.5 \%$ in 61 patients. ORR was at least 3.5fold higher in patients with LAG-3 expression in at least $1 \%$ of tumor-associated immune cells within the tumor margin $(\mathrm{n}=33)$ than that in the patients with less than 1\% LAG-3 expression ( $\mathrm{n}=22)$ (18\% and 5\%, respectively) ${ }^{109}$ LAG525 in combination with anti-PD-1 Ab (spartalizumab) exhibited a durable response in $9.9 \%$ of patients $(n=121)$ with a variety of solid tumors, including mesothelioma (two of eight patients) and triple-negative breast cancer (two of five patients) in phase I/II study (NCT02460224). ${ }^{110}$ The precise mechanism of the function of such anti-LAG-3 Abs remains to be investigated. Especially, the examination of target cells in anti-LAG-3 therapy and elucidation of the mechanisms of synergy between anti-LAG-3 and anti-PD-1 therapies have garnered interest and are required for the rational design of anti-LAG-3 therapy with maximum efficacy and minimal adverse effects.

Other LAG-3-targeting agents have also been tested for cancer treatment. IMP321, a soluble recombinant fusion protein comprising the extracellular region of LAG-3 and the Fc region of IgG, has been reported to activate antigenpresenting cells by transducing a reverse signal via MHCII, 
resulting in the enhanced production of IL-12 and TNF and the upregulation of CD80 and CD86. ${ }^{111} 112$ IMP321 demonstrated only minimal or modest efficacy as monotherapy or in combination with other therapies in clinical trials conducted so far. ${ }^{113-116}$ The details of the reverse signal via MHCII remain unknown and require careful investigation.

The development of anti-LAG-3 depleting $\mathrm{Ab}$ (GSK2831781) and agonistic Ab (IMP761) as potential therapeutic agents in the treatment of autoimmune diseases has also been reported. ${ }^{90}{ }^{91}$ Although these Abs aim to remove or suppress pathogenic $\mathrm{T}$ cells, they may also deplete or suppress $\mathrm{T}_{\text {reg }}$ cells. Further studies elucidating the functions of such Abs together with the biological properties of LAG-3 are expected to help advance their development by providing the rationale for their use.

\section{CONCLUSIONS}

As checkpoint immunotherapies targeting inhibitory coreceptors PD-1 and CTLA-4 revolutionized cancer treatment, LAG-3 is expected to be a highly promising target in cancer therapies. However, our understanding of LAG-3 is still very limited and many fundamental questions remain unanswered. The signaling mechanism of LAG-3 is unknown and the ligands of LAG-3 are perplexing. LAG-3 is expressed on a variety of cell types. However, the function of LAG-3 and the effect of LAG-3 blockade in each type of cells have not been elucidated. We also need to examine the functional differences, redundancies, and co-operations of LAG-3 and other coreceptors. By elucidating the functional properties of LAG-3 more in detail, we can rationally design LAG-3targeted therapies for various diseases, such as cancer, autoimmunity, and infection.

Acknowledgements We thank the lab members for helpful discussions.

Contributors TM, DS, IMO, and TO wrote the review.

Funding This work was supported in part by a Grant-in-Aid from the Japan Society for the Promotion of Science (JP18H05417, JP18K19453, JP19H01029, JP19H03423, JP19K16694).

Competing interests None declared.

Patient consent for publication Not required.

Provenance and peer review Commissioned; externally peer reviewed.

Open access This is an open access article distributed in accordance with the Creative Commons Attribution Non Commercial (CC BY-NC 4.0) license, which permits others to distribute, remix, adapt, build upon this work non-commercially, and license their derivative works on different terms, provided the original work is properly cited, appropriate credit is given, any changes made indicated, and the use is non-commercial. See http://creativecommons.org/licenses/by-nc/4.0/.

\section{ORCID iDs}

Takumi Maruhashi http://orcid.org/0000-0003-2944-1611

Daisuke Sugiura http://orcid.org/0000-0002-3865-3889

II-mi Okazaki http://orcid.org//0000-0001-8644-0100

Taku Okazaki http://orcid.org/0000-0003-4790-1925

\section{REFERENCES}

1 Okazaki T, Chikuma S, Iwai Y, et al. A rheostat for immune responses: the unique properties of $\mathrm{PD}-1$ and their advantages for clinical application. Nat Immunol 2013;14:1212-8.
2 Ribas A, Wolchok JD. Cancer immunotherapy using checkpoint blockade. Science 2018;359:1350-5.

3 Topalian SL, Taube JM, Anders RA, et al. Mechanism-Driven biomarkers to guide immune checkpoint blockade in cancer therapy. Nat Rev Cancer 2016;16:275-87.

4 Sharpe $\mathrm{AH}$, Pauken KE. The diverse functions of the PD1 inhibitory pathway. Nat Rev Immunol 2018;18:153-67.

5 Sun C, Mezzadra R, Schumacher TN. Regulation and function of the PD-L1 checkpoint. Immunity 2018;48:434-52.

6 Andrews LP, Yano H, Vignali DAA. Inhibitory receptors and ligands beyond PD-1, PD-L1 and CTLA-4: breakthroughs or backups. Nat Immunol 2019;20:1425-34.

7 Triebel F. LAG-3: a regulator of T-cell and DC responses and its use in therapeutic vaccination. Trends Immunol 2003;24:619-22.

8 Triebel F, Jitsukawa S, Baixeras E, et al. LAG-3, a novel lymphocyte activation gene closely related to CD4. J Exp Med 1990;171:1393-405.

9 Crise B, Rose JK. Identification of palmitoylation sites on CD4, the human immunodeficiency virus receptor. $\mathrm{J}$ Biol Chem 1992;267:13593-7.

10 Shaw AS, Chalupny J, Whitney JA, et al. Short related sequences in the cytoplasmic domains of CD4 and CD8 mediate binding to the amino-terminal domain of the p56lck tyrosine protein kinase. $\mathrm{Mol}$ Cell Biol 1990;10:1853-62.

11 Turner JM, Brodsky MH, Irving BA, et al. Interaction of the unique $\mathrm{N}$-terminal region of tyrosine kinase p56lck with cytoplasmic domains of CD4 and CD8 is mediated by cysteine motifs. Cell 1990;60:755-65.

12 Huard B, Mastrangeli R, Prigent P, et al. Characterization of the major histocompatibility complex class II binding site on LAG-3 protein. Proc Natl Acad Sci U S A 1997;94:5744-9.

13 Workman CJ, Dugger KJ, Vignali DAA. Cutting edge: molecular analysis of the negative regulatory function of lymphocyte activation gene-3. J Immunol 2002;169:5392-5.

14 Baixeras E, Huard B, Miossec C, et al. Characterization of the lymphocyte activation gene 3 -encoded protein. A new ligand for human leukocyte antigen class II antigens. J Exp Med 1992;176:327-37.

15 Kouo T, Huang L, Pucsek AB, et al. Galectin-3 shapes antitumo immune responses by suppressing CD8+ T cells via LAG-3 and inhibiting expansion of plasmacytoid dendritic cells. Cancer Immunol Res 2015;3:412-23.

16 Xu F, Liu J, Liu D, et al. Lsectin expressed on melanoma cells promotes tumor progression by inhibiting antitumor T-cell responses. Cancer Res 2014;74:3418-28.

17 Li N, Wang Y, Forbes K, et al. Metalloproteases regulate Tcell proliferation and effector function via LAG-3. Embo $J$ 2007;26:494-504.

18 Annunziato F, Manetti R, Tomasévic I, et al. Expression and release of LAG-3-encoded protein by human CD4 ${ }^{+} \mathrm{T}$ cells are associated with IFN- $\gamma$ production. Faseb J 1996;10:769-76.

19 Okazaki T, Okazaki IM, Wang J, et al. PD-1 and LAG-3 inhibitory co-receptors act synergistically to prevent autoimmunity in mice. $J$ Exp Med 2011;208:395-407.

20 Maeda TK, Sugiura D, Okazaki IM, et al. Atypical motifs in the cytoplasmic region of the inhibitory immune co-receptor LAG-3 inhibit T cell activation. J Biol Chem 2019;294:6017-26.

21 Blackburn SD, Shin H, Haining WN, et al. Coregulation of CD8+ T cell exhaustion by multiple inhibitory receptors during chronic viral infection. Nat Immunol 2009;10:29-37.

22 Tian X, Zhang A, Qiu C, et al. The upregulation of LAG-3 on T cells defines a subpopulation with functional exhaustion and correlates with disease progression in HIV-infected subjects. J Immunol 2015;194:3873-82.

23 Phillips BL, Mehra S, Ahsan MH, et al. LAG3 expression in active Mycobacterium tuberculosis infections. Am J Pathol 2015;185:820-33.

24 Butler NS, Moebius J, Pewe LL, et al. Therapeutic blockade of PDL1 and LAG-3 rapidly clears established blood-stage Plasmodium infection. Nat Immunol 2011;13:188-95.

25 Chen N, Liu Y, Guo Y, et al. Lymphocyte activation gene 3 negatively regulates the function of intrahepatic hepatitis $C$ virusspecific CD8+ T cells. J Gastroenterol Hepatol 2015;30:1788-95.

26 Dong Y, Li X, Zhang L, et al. CD4 ${ }^{+} \mathrm{T}$ cell exhaustion revealed by high PD-1 and LAG-3 expression and the loss of helper T cell function in chronic hepatitis B. BMC Immunol 2019;20:27.

27 Erickson JJ, Rogers MC, Tollefson SJ, et al. Multiple inhibitory pathways contribute to lung CD8+ T cell impairment and protect against immunopathology during acute viral respiratory infection. $J$ Immunol 2016;197:233-43. 
28 Roy S, Coulon P-G, Srivastava R, et al. Blockade of LAG-3 Immune Checkpoint Combined With Therapeutic Vaccination Restore the Function of Tissue-Resident Anti-viral CD8 ${ }^{+} \mathrm{T}$ Cells and Protect Against Recurrent Ocular Herpes Simplex Infection and Disease. Front Immunol 2018;9:2922.

29 Cook KD, Whitmire JK. LAG-3 confers a competitive disadvantage upon antiviral CD8+ T cell responses. J Immunol 2016;197:119-27.

30 Richter K, Agnellini P, Oxenius A. On the role of the inhibitory receptor LAG-3 in acute and chronic LCMV infection. Int Immunol 2010;22:13-23.

31 Demeure CE, Wolfers J, Martin-Garcia N, et al. T lymphocytes infiltrating various tumour types express the $\mathrm{MHC}$ class II ligand lymphocyte activation gene-3 (LAG-3): role of LAG-3/MHC class II interactions in cell-cell contacts. Eur J Cancer 2001;37:1709-18.

32 Gandhi MK, Lambley E, Duraiswamy J, et al. Expression of LAG-3 by tumor-infiltrating lymphocytes is coincident with the suppression of latent membrane antigen-specific CD8+ T-cell function in Hodgkin lymphoma patients. Blood 2006;108:2280-9.

33 Li F-J, Zhang Y, Jin G-X, et al. Expression of LAG-3 is coincident with the impaired effector function of HBV-specific CD8(+) T cell in HCC patients. Immunol Lett 2013;150:116-22.

34 Matsuzaki J, Gnjatic S, Mhawech-Fauceglia P, et al. TumorInfiltrating NY-ESO-1-specific CD8+ T cells are negatively regulated by LAG-3 and PD-1 in human ovarian cancer. Proc Natl Acad Sci U $S$ A 2010;107:7875-80.

35 Thommen DS, Schreiner J, Müller P, et al. Progression of lung cancer is associated with increased dysfunction of $T$ cells defined by coexpression of multiple inhibitory receptors. Cancer Immunol Res 2015;3:1344-55.

36 Yang Z-Z, Kim HJ, Villasboas JC, et al. Expression of LAG-3 defines exhaustion of intratumoral PD- $1^{+}$T cells and correlates with poor outcome in follicular lymphoma. Oncotarget 2017;8:61425-39.

37 Bruniquel D, Borie N, Hannier S, et al. Regulation of expression of the human lymphocyte activation gene-3 (LAG-3) molecule, a ligand for MHC class II. Immunogenetics 1998;48:116-24.

38 Sun $\mathrm{H}$, Sun C, Xiao W. Expression regulation of co-inhibitory molecules on human natural killer cells in response to cytokine stimulations. Cytokine 2014;65:33-41.

39 Huang C-T, Workman CJ, Flies D, et al. Role of LAG-3 in regulatory T cells. Immunity 2004;21:503-13.

40 Li MO, Rudensky AY. T cell receptor signalling in the control of regulatory T cell differentiation and function. Nat Rev Immunol 2016:16:220-33.

41 Zhang Q, Chikina M, Szymczak-Workman AL, et al. LAG3 limits regulatory $T$ cell proliferation and function in autoimmune diabetes. Sci Immunol 2017;2. doi:10.1126/sciimmunol.aah4569. [Epub ahead of print: 31 Mar 2017].

42 Durham NM, Nirschl CJ, Jackson CM, et al. Lymphocyte activation gene 3 (LAG-3) modulates the ability of CD4 T-cells to be suppressed in vivo. PLoS One 2014;9:e109080.

43 Sega El, Leveson-Gower DB, Florek M, et al. Role of lymphocyte activation gene-3 (Lag-3) in conventional and regulatory T cell function in allogeneic transplantation. PLoS One 2014;9:e86551.

44 Gagliani N, Magnani CF, Huber S, et al. Coexpression of CD49b and LAG-3 identifies human and mouse T regulatory type 1 cells. Nat Med 2013;19:739-46.

45 Okamura T, Fujio K, Shibuya M, et al. CD4+CD25-LAG3+ regulatory T cells controlled by the transcription factor Egr-2. Proc Natl Acad Sci U S A 2009;106:13974-9.

46 Okamura T, Sumitomo S, Morita K, et al. TGF- 33 -expressing CD4+CD25(-)LAG3+ regulatory T cells control humoral immune responses. Nat Commun 2015;6:6329.

47 Lino AC, Dang VD, Lampropoulou V, et al. LAG-3 inhibitory receptor expression identifies immunosuppressive natural regulatory plasma cells. Immunity 2018;49:120-33.

48 Chen J, López-Moyado IF, Seo H, et al. Nr4A transcription factors limit CAR T cell function in solid tumours. Nature 2019;567:530-4.

49 Khan O, Giles JR, McDonald S, et al. TOX transcriptionally and epigenetically programs $\mathrm{CD} 8^{+} \mathrm{T}$ cell exhaustion. Nature 2019;571:211-8.

50 Liu X, Wang Y, Lu H, et al. Genome-Wide analysis identifies Nr4a1 as a key mediator of T cell dysfunction. Nature 2019;567:525-9.

51 Martinez GJ, Pereira RM, Äijö T, et al. The transcription factor NFAT promotes exhaustion of activated CD8 $8^{+} \mathrm{T}$ cells. Immunity 2015;42:265-78.

52 Seo H, Chen J, González-Avalos E, et al. TOX and TOX2 transcription factors cooperate with NR4A transcription factors to impose CD8 ${ }^{+} \mathrm{T}$ cell exhaustion. Proc Natl Acad Sci U S A 2019;116:12410-5.
$53 \mathrm{Wu}$ J, Zhang H, Shi X, et al. Ablation of Transcription Factor IRF4 Promotes Transplant Acceptance by Driving Allogenic CD4 ${ }^{+} \mathrm{T}$ Cell Dysfunction. Immunity 2017;47:1114-28.

54 Man K, Gabriel SS, Liao Y, et al. Transcription Factor IRF4 Promotes $\mathrm{CD}^{+} \mathrm{T}$ Cell Exhaustion and Limits the Development of Memory-like T Cells during Chronic Infection. Immunity 2017;47:1129-41.

55 Scott AC, Dündar F, Zumbo P, et al. Tox is a critical regulator of tumour-specific T cell differentiation. Nature 2019;571:270-4.

56 Shin $\mathrm{H}$, Blackburn SD, Intlekofer AM, et al. A role for the transcriptional repressor Blimp-1 in CD8(+) T cell exhaustion during chronic viral infection. Immunity 2009;31:309-20.

57 Kao C, Oestreich KJ, Paley MA, et al. Transcription factor T-bet represses expression of the inhibitory receptor PD-1 and sustains virus-specific CD8+ T cell responses during chronic infection. Nat Immunol 2011:12:663-71.

58 Rudd CE, Chanthong K, Taylor A. Small molecule inhibition of GSK-3 specifically inhibits the transcription of inhibitory coreceptor LAG-3 for enhanced anti-tumor immunity. Cell Rep 2020;30:2075-82

59 Bae J, Lee SJ, Park C-G, et al. Trafficking of LAG-3 to the surface on activated $T$ cells via its cytoplasmic domain and protein kinase $C$ signaling. J Immunol 2014;193:3101-12.

60 Li N, Workman CJ, Martin SM, et al. Biochemical analysis of the regulatory $T$ cell protein lymphocyte activation gene-3 (LAG-3; CD223). J Immunol 2004;173:6806-12.

61 Tian D, Yang L, Wang S, et al. Double negative T cells mediate Lag3-dependent antigen-specific protection in allergic asthma. Nat Commun 2019;10:4246.

62 Denning TL, Granger SW, Granger S, et al. Mouse TCRalphabeta+CD8alphaalpha intraepithelial lymphocytes express genes that down-regulate their antigen reactivity and suppress immune responses. J Immunol 2007;178:4230-9.

63 Fahrer AM, Konigshofer Y, Kerr EM, et al. Attributes of gammadelta intraepithelial lymphocytes as suggested by their transcriptional profile. Proc Natl Acad Sci U S A 2001;98:10261-6.

64 Workman CJ, Rice DS, Dugger KJ, et al. Phenotypic analysis of the murine CD4-related glycoprotein, CD223 (LAG-3). Eur J Immunol 2002;32:2255-63.

65 Byun H-J, Jung W-W, Lee D-S, et al. Proliferation of activated CD1d-restricted NKT cells is down-modulated by lymphocyte activation gene-3 signaling via cell cycle arrest in S phase. Cell Biol Int 2007;31:257-62.

66 Miyazaki T, Dierich A, Benoist C, et al. LAG-3 is not responsible for selecting Thelper cells in CD4-deficient mice. Int Immunol 1996;8:725-9.

67 Workman CJ, Wang Y, El Kasmi KC, et al. LAG-3 regulates plasmacytoid dendritic cell homeostasis. J Immunol 2009;182:1885-91.

68 Kisielow M, Kisielow J, Capoferri-Sollami G, et al. Expression of lymphocyte activation gene 3 (LAG-3) on B cells is induced by $T$ cells. Eur J Immunol 2005;35:2081-8.

69 Mao X, Ou MT, Karuppagounder SS, et al. Pathological $\alpha$-synuclein transmission initiated by binding lymphocyte-activation gene 3 . Science 2016;353. doi:10.1126/science.aah3374.

70 Huard B, Prigent P, Tournier M, et al. CD4/major histocompatibility complex class II interaction analyzed with CD4- and lymphocyte activation gene-3 (LAG-3)-Ig fusion proteins. Eur J Immunol 1995;25:2718-21.

71 Maruhashi T, Okazaki IM, Sugiura D, et al. LAG-3 inhibits the activation of $\mathrm{CD}^{+} \mathrm{T}$ cells that recognize stable pMHCll through its conformation-dependent recognition of pMHCII. Nat Immunol 2018:19:1415-26.

72 Neefjes J, Jongsma MLM, Paul P, et al. Towards a systems understanding of $\mathrm{MHC}$ class I and $\mathrm{MHC}$ class II antigen presentation. Nat Rev Immunol 2011;11:823-36.

73 Liu W, Tang L, Zhang G, et al. Characterization of a novel C-type lectin-like gene, LSECtin: demonstration of carbohydrate binding and expression in sinusoidal endothelial cells of liver and lymph node. J Biol Chem 2004;279:18748-58.

74 Ruvolo PP. Galectin 3 as a guardian of the tumor microenvironment. Biochim Biophys Acta 2016;1863:427-37.

75 Wang J, Sanmamed MF, Datar I, et al. Fibrinogen-Like protein 1 is a major immune inhibitory ligand of LAG-3. Cell 2019;176:334-47.

76 Nishide M, Kumanogoh A. The role of semaphorins in immune responses and autoimmune rheumatic diseases. Nat Rev Rheumatol 2018;14:19-31.

77 Takeda K, Nakamura A. Regulation of immune and neural function via leukocyte Ig-like receptors. J Biochem 2017;162:73-80.

78 louzalen N, Andreae S, Hannier S, et al. LAP, a lymphocyte activation gene-3 (LAG-3)-associated protein that binds to a repeated EP motif in the intracellular region of LAG-3, may 
participate in the down-regulation of the CD3/TCR activation pathway. Eur J Immunol 2001;31:2885-91.

79 Nishimura $\mathrm{H}$, Nose M, Hiai H, et al. Development of lupus-like autoimmune diseases by disruption of the PD-1 gene encoding an ITIM motif-carrying immunoreceptor. Immunity 1999;11:141-51.

80 Tivol EA, Borriello F, Schweitzer AN, et al. Loss of CTLA-4 leads to massive lymphoproliferation and fatal multiorgan tissue destruction, revealing a critical negative regulatory role of CTLA-4. Immunity 1995;3:541-7.

81 Waterhouse P, Penninger JM, Timms E, et al. Lymphoproliferative disorders with early lethality in mice deficient in CTLA-4. Science 1995;270:985-8.

82 Martins F, Sofiya L, Sykiotis GP, et al. Adverse effects of immunecheckpoint inhibitors: epidemiology, management and surveillance. Nat Rev Clin Oncol 2019;16:563-80.

84 Okazaki T, Okazaki IM. Stimulatory and inhibitory Co-signals in autoimmunity. Adv Exp Med Biol 2019;1189:213-32.

84 Postow MA, Sidlow R, Hellmann MD. Immune-Related adverse events associated with immune checkpoint blockade. N Engl J Med 2018;378:158-68.

85 Bettini M, Szymczak-Workman AL, Forbes K, et al. Cutting edge: accelerated autoimmune diabetes in the absence of LAG-3. $J$ Immunol 2011;187:3493-8.

86 Woo S-R, Turnis ME, Goldberg MV, et al. Immune inhibitory molecules LAG-3 and PD-1 synergistically regulate T-cell function to promote tumoral immune escape. Cancer Res 2012;72:917-27.

87 Jha V, Workman CJ, McGaha TL, et al. Lymphocyte activation Gene-3 (LAG-3) negatively regulates environmentally-induced autoimmunity. PLoS One 2014;9:e104484.

88 Kadowaki A, Miyake S, Saga R, et al. Gut environment-induced intraepithelial autoreactive CD4(+) T cells suppress central nervous system autoimmunity via LAG-3. Nat Commun 2016;7:11639.

89 Kim D, Le HT, Nguyen QT, et al. Cutting edge: IL-27 attenuates autoimmune neuroinflammation via regulatory $\mathrm{T}$ Cell/Lag3Dependent but IL-10-Independent mechanisms in vivo. J Immunol 2019;202:1680-5.

90 Poirier N, Haudebourg T, Brignone C, et al. Antibody-mediated depletion of lymphocyte-activation gene-3 (LAG-3(+))-activated T lymphocytes prevents delayed-type hypersensitivity in non-human primates. Clin Exp Immunol 2011;164:265-74.

91 Angin M, Brignone C, Triebel F. A LAG-3-Specific agonist antibody for the treatment of $T$ cell-induced autoimmune diseases. $J$ Immunol 2020;204:810-8.

92 Camisaschi C, Casati C, Rini F, et al. LAG-3 expression defines a subset of CD4(+)CD25(high)Foxp3(+) regulatory T cells that are expanded at tumor sites. $J$ Immunol 2010;184:6545-51.

93 Wei T, Zhang J, Qin Y, et al. Increased expression of immunosuppressive molecules on intratumoral and circulating regulatory T cells in non-small-cell lung cancer patients. $\mathrm{Am} \mathrm{J}$ Cancer Res 2015:5:2190-201.

94 Chen J, Chen Z. The effect of immune microenvironment on the progression and prognosis of colorectal cancer. Med Oncol 2014;31:82.

95 Giraldo NA, Becht E, Pagès F, et al. Orchestration and prognostic significance of immune checkpoints in the microenvironment of primary and metastatic renal cell cancer. Clin Cancer Res 2015;21:3031-40

96 Deng W-W, Mao L, Yu G-T, et al. LAG-3 confers poor prognosis and its blockade reshapes antitumor response in head and neck squamous cell carcinoma. Oncoimmunology 2016;5:e1239005.

$97 \mathrm{He} \mathrm{Y,} \mathrm{Yu} \mathrm{H,} \mathrm{Rozeboom} \mathrm{L,} \mathrm{et} \mathrm{al.} \mathrm{LAG-3} \mathrm{protein} \mathrm{expression} \mathrm{in} \mathrm{non-}$ small cell lung cancer and its relationship with PD-1/PD-L1 and tumor-infiltrating lymphocytes. J Thorac Oncol 2017;12:814-23.

98 Burugu S, Gao D, Leung S, et al. LAG-3+ tumor infiltrating lymphocytes in breast cancer: clinical correlates and association with PD-1/PD-L1+ tumors. Ann Oncol 2017;28:2977-84.
99 Keane C, Law SC, Gould C, et al. LAG3: a novel immune checkpoint expressed by multiple lymphocyte subsets in diffuse large B-cell lymphoma. Blood Adv 2020;4:1367-77.

100 Que Y, Fang Z, Guan Y, et al. LAG-3 expression on tumor-infiltrating T cells in soft tissue sarcoma correlates with poor survival. Cancer Biol Med 2019;16:331-40.

101 Grosso JF, Kelleher CC, Harris TJ, et al. LAG-3 regulates CD8+ T cell accumulation and effector function in murine self- and tumortolerance systems. J Clin Invest 2007;117:3383-92.

102 Goding SR, Wilson KA, Xie Y, et al. Restoring immune function of tumor-specific CD4+ T cells during recurrence of melanoma. $J$ Immunol 2013;190:4899-909.

103 Huang R-Y, Eppolito C, Lele S, et al. LAG3 and PD1 co-inhibitory molecules collaborate to limit CD8+ T cell signaling and dampen antitumor immunity in a murine ovarian cancer model. Oncotarget 2015;6:27359-77.

104 Wierz M, Pierson S, Guyonnet L, et al. Dual PD1/LAG3 immune checkpoint blockade limits tumor development in a murine model of chronic lymphocytic leukemia. Blood 2018;131:1617-21.

105 LaMotte-Mohs R, Shah K, Smith D, et al. MGD013, a bispecific PD-1 X LAG-3 dual affinity re-targeting (DART $®$ ) protein with T-cell immunomodulatory activity for cancer treatment. Cancer Res 2016;76.

106 Savitsky D, Ward R, Riordan C, et al. INCAGN02385 is an antagonist antibody targeting the co-inhibitory receptor LAG-3 for the treatment of human malignancies. Cancer Res 2018;78.

107 Zettl M, Wurm M, Schaaf O, et al. Characterization of the LAG-3 targeting antibody $\mathrm{BI} 754111$ in monotherapy and in combination with the anti-PD-1 antibody RI 754091. Can Res 2018;78.

108 Kraman M, Faroudi M, Allen NL, et al. FS118, a bispecific antibody targeting LAG-3 and PD-L1, enhances T-cell activation resulting in potent antitumor activity. Clin Cancer Res 2020;26:3333-44.

109 Ascierto P, Bono P, Bhatia S, et al. Efficacy of BMS-986016, a monoclonal antibody that targets lymphocyte activation gene-3 (LAG-3), in combination with PD-L1 therapy (MEL prior io) in all-comer and biomarker-enriched populations. Ann Oncol;217:v605-49.

110 Hong DS, Schoffski P, Calvo A, et al. Phase I/II study of LAG525 \pm spartalizumab (PDR001) in patients (PTS) with advanced malignancies. JCO 2018;36:3012.

111 Avice MN, Sarfati M, Triebel F, et al. Lymphocyte activation gene-3, a MHC class II ligand expressed on activated T cells, stimulates TNF-alpha and IL-12 production by monocytes and dendritic cells. J Immunol 1999;162:2748-53.

112 Buisson S, Triebel F. Mhc class II engagement by its ligand LAG-3 (CD223) leads to a distinct pattern of chemokine and chemokine receptor expression by human dendritic cells. Vaccine 2003;21:862-8.

113 Brignone C, Gutierrez M, Mefti F, et al. First-Line chemoimmunotherapy in metastatic breast carcinoma: combination of paclitaxel and IMP321 (LAG-3lg) enhances immune responses and antitumor activity. J Transl Med 2010;8:71.

114 Legat A, Maby-El Hajjami H, Baumgaertner P, et al. Vaccination with LAG-3lg (IMP321) and Peptides Induces Specific CD4 and CD8 T-Cell Responses in Metastatic Melanoma Patients--Report of a Phase I/Ila Clinical Trial. Clin Cancer Res 2016;22:1330-40.

115 Romano E, Michielin O, Voelter V, et al. Mart-1 peptide vaccination plus IMP321 (LAG-3lg fusion protein) in patients receiving autologous PBMCs after lymphodepletion: results of a phase I trial. $J$ Transl Med 2014;12:97.

116 Wang-Gillam A, Plambeck-Suess S, Goedegebuure P, et al. A phase I study of IMP321 and gemcitabine as the front-line therapy in patients with advanced pancreatic adenocarcinoma. Invest New Drugs 2013;31:707-13. 